\title{
EFICIENCIA ECONOMICA DE LA INVERSION PÚBLICA FINANCIADA CON RECURSOS DEL CANON Y REGALIAS MINERAS EN EL PERU
}

\author{
ECONOMIC EFFICIENCY OF PUBLIC INVESTMENT FINANCED WITH CANON \\ RESOURCES AND MINING ROYALTIES IN PERU
}

\author{
${ }^{1} J u a n$ Magallanes Díaz
}

\begin{abstract}
Resumen
Las rentas generadas por la explotación de los recursos naturales deberían ser orientadas a inversiones que promuevan nuevas economías en el largo plazo, y deberían reflejarse en mejoras en el bienestar de la sociedad en un espacio económico. El presente documento tiene como propósito analizar la eficiencia relativa del gasto de inversión pública financiado con recursos de la renta minera en cinco principales regiones mineras del Perú para el periodo 2004-2015. Se utiliza la metodología de Free Disposal Hull (FDH), donde se procesan 7 indicadores de desempeño económico y social así como de un indicador de recursos. Se concluye que no se han logrado un desempeño homogéneo en su bienestar, regiones que han tenido menos recursos financieros ha sido más eficientes en lograr mejores resultados económicos y sociales, en comparación a otras regiones con más recursos financieros han conseguido relativamente bajo desempeño económico y social.
\end{abstract}

Palabras clave: inversión pública, canon minero, eficiencia del gasto público.

\begin{abstract}
The income generated by the exploitation of natural resources should be targeted to investments to promote new economies in the long term, and should be reflected in improvements in the welfare of society in an economic space. This paper aims to analyze the relative efficiency of public investment expenditure financed with mining income in five major mining regions of Peru 2004-2015. The methodology of Free Disposal Hull (FDH), with 7 indicators of economic and social performance as well as an indicator of resources used are processed. It is concluded that has not been achieved homogeneous performance in their welfare, regions that have fewer financial resources has been more efficient in achieving better economic and social outcomes, compared to other regions with more financial resources have achieved relatively low economic and social performance.
\end{abstract}

Keywords: public investment, mining royalties, efficiency of public spending.

\section{Introducción}

En los últimos diez años, la economía peruana ha venido mostrando un crecimiento sólido y estable dentro de la región de Sudamérica, no obstante ello, no logra superar las grandes asimetrías productivas y desigualdades sociales entre las diferentes regiones del país. Este crecimiento económico se ha sustentado principalmente en la explotación de sus recursos naturales, modelo muy típico desde la época virreinal y la actual República. $\mathrm{Y}$ es justamente en aquellas regiones generadoras de rentas mineras, gasíferas, petroleras, pesqueras y forestales donde la estadística oficial reporta esta severas diferencias.

Las rentas generadas de las explotación minera constituyen fuente del canon y regalías mineras que son transferidas en una gran proporción desde el gobierno central a los gobiernos regionales, provinciales y distritales, con el propósito que participen estas instancias de gobiernos en las decisiones del destino y gestión de estos recursos, aunque la legislación es clara en que el uso de estos recursos es para el desarrollo de impacto regional, y que en buena cuenta debe reflejarse en indicadores de resultado económico y social en cada una de las regiones.

Según la Economía del Bienestar, un gasto tiene sentido económico si obtiene el máximo nivel de bienestar social, para alcanzarlo este gasto debe ser eficiente en términos de los recursos empleados y equitativos en término de resultados para cada miembro de la sociedad, el resultado debe cubrir los costos y obtener un saldo positivo para luego distribuirlo a los miembros de la sociedad.

La presente investigación procesa datos de recursos del canon y regalías mineras transferidas a cada región minera y los compara con indicadores de desempeño o resultados económicos y sociales, de tal forma que se trata aquí de dar cuenta resultados de eficiencia en el gasto en

\footnotetext{
${ }^{1}$ Economista, Mg. Sc. en Economía Agrícola y Desarrollo Rural, profesor principal del Departamento Académico de Economía y Planificación de la
} Universidad Nacional Agraria La Molina, Lima, Perú. E-mail: jmagallanes@lamolina.edu.pe 
inversión pública desde el estado descentralizado en las regiones políticas del país, no sin eximir responsabilidades de la iniciativa privada, principal explotador del recurso natural.

Por ello, el objetivo de la presente investigación es determinar la eficiencia relativa del gasto de inversión pública en las principales regiones mineras del Perú financiado con recursos del canon y regalías mineras, estimando una frontera de posibilidades de producción a través de la metodología de Free Disposal Hull (FDH), mediante el enfoque de pruebas no paramétricas para el periodo del 2004-2015. La muestra está conformada por las 5 principales regiones mineras del País que cuentan con la información existente para poder ser representadas dentro del marco de este análisis.

\section{Revisión de literatura}

La frontera de posibilidades de producción (FPP), se define a las cantidades máximas de producción que puede obtener una economía, dados sus conocimientos tecnológicos y la cantidad disponible de insumos, La FPP tiene la capacidad de representar una variedad de bienes y servicios disponibles para una sociedad, y su aplicación fundamental es para indicar si la economía opera eficientemente ubicándose en la misma frontera. (Samuelson P., Nordhaus W., 2010)

Actualmente la FPP se emplea en diferentes tipos de análisis para medir eficiencia técnica en los diferentes tipos de sectores como salud, agricultura, educación, social entre otros, los análisis se realizan con el fin de conocer la productividad de los recursos empleados en cada sector dado, los resultados obtenidos permiten tomar decisiones como incrementar los insumos o mejorar la administración de los recursos a fin de alcanzar un mayor nivel de eficiencia.

En las distintas economías a nivel mundial como el caso de África, Asia, Europa y América están empleando el análisis de la FPP para medir la eficiencia relativa a través de indicadores y su nivel de gasto público recurrido en distintas economías de su región para poder ejecutar medidas de política económica y alcanzar a la economía más eficiente o benchmarking (Gupta S., Verhoeven M., 1999; Afonso A., Schuknecht L., Vito T., 2003; Afonso A., Schuknecht L., Vito T., 2006; Machado R., 2006).

En la actualidad, se presentan dificultades sobre determinar el verdadero valor que tiene la ejecución del gasto público en diferentes usos al ofrecer bienes o al brindar un servicio, debido a que no existe un medio eficaz para que los consumidores puedan expresar sus preferencias, por esa razón se recurre a diferentes medios, uno de ellos son los impuestos segmentando a las personas más ricas y pobres constituyéndose un cobro diferenciado a través de un sistema impositivo.

Se debe mencionar que otro problema latente son las decisiones que se toman de manera colectiva, esto se observa cuando deben decidir dónde invertir el gasto público, valorando las preferencias de los potenciales ciudadanos beneficiados que brindan preferencias diferentes sobre el uso en la ejecución del gasto público, este problema de la conciliación se debe solucionar mediante un proceso de votación que es insuficiente, porque se presenta la paradoja del voto donde exactamente no existe un claro dominador sobre la decisión que se debería ejecutar el gasto público (Arrow K., 1974).

Para poder comprender las decisiones colectivas, debemos centrar la atención del votante mediano (que son los votantes que determinan la decisión sobre la elección de la ejecución del gasto público), y en la forma que le impacta un determinado programa como la posibilidad de deducir los impuestos de los gobiernos regionales sobre la renta y sobre el patrimonio del impuesto predial, sin embargo existen otros factores que no le permiten obtener el resultado que desean y se debe analizar diversas consideraciones en los procesos políticos.

Cabe precisar que existen posturas sobre la ejecución del gasto público como el teorema de la imposibilidad de Arrow quien demostró que no existía ninguna regla de elección que satisfaga a todas las características deseadas de la sociedad, sugiere que esto es posible a menos que concedamos a una persona poderes dictatoriales, no podemos esperar que el Gobierno actúe con el mismo grado de coherencia y racionalidad que una persona, esto se debe a que Arrow señala que no es posible sumar las preferencias de los diferentes individuos para satisfacer todas las características que debe tener un mecanismo de decisión, se ha demostrado que no existe, en general, ningún sistema de votación en el que los individuos siempre prefieran votar de acuerdo con sus verdaderas preferencias (Arrow K., 1974).

Otra de las posturas de la Teoría del Gasto Público menciona la influencia aparentemente desproporcionada de algunos pequeños grupos de interés para tomar decisiones sobre la elección del gasto público, esto se observa claramente dentro de la política y se tendrán perdedores, pero su pérdida debe ser menor para que se obtenga un mejor resultado. (Becker G., 1983).

En cuanto a los aspectos de la medición, uno de los métodos utilizados es el análisis coste beneficio, enfoque que permite tomar constantemente decisiones sobre la conveniencia de emprender determinadas inversiones, primero se debe identificar el conjunto de proyectos posibles que debe examinarse, reconocer las consecuencias de estas posibilidades, luego asignarles un valor a cada uno de los factores y resultados obtenidos y por ultimo sumando los costes con los beneficios para hallar la rentabilidad total del proyecto teniendo en cuenta el costo de oportunidad es decir el rendimiento de los fondos públicos invertidos en otra parte. Este enfoque no es lo suficientemente claro en concluir sobre la eficiencia de la inversión en los resultados sociales esperados.

En la presente investigación haremos uso de las técnicas no paramétricas, las que se emplean para la medición de la eficiencia en el sector público. Para esto se debe tener 
información sobre el nivel de producto o resultado y los insumos necesarios para alcanzar tal o tales niveles del producto, para este estudio el producto son considerados los índices socioeconómicos y los insumos son el gasto de inversión pública por regiones mineras dentro de cada área a evaluar y por medio de la frontera se pueda visualizar la eficiencia de cada región analizada, entre los modelos más destacados son el Análisis Envolvente de Datos (DEA) y Free Disposal Hull (FDH).

Ambas técnicas permiten estimar los puntajes de eficiencia mediante la orientación al insumo u orientación al producto, los investigadores que las han aplicado sugieren que estas son más adecuadas para el análisis de la eficiencia del sector público (Afonso A. y St. Aubyn, 2004. Ahn T.; Ahn Charnes W. y Cooper W., 1988. Asmad U.; Palomino D.; Tam M. y Zambrano G., 2004. Banker R.; Charnes A. y Cooper W., 1984). Las razones son diversas como que no requieren la especificación de precios a los que se valoren los costos o beneficios de la producción, trabajan con multiplicidad de productos, no requieren supuestos sobre la tecnología de la producción, la cual afecta a los métodos de técnicas paramétricas.

La diferencia del DEA y el FDH es que el FDH en este tipo de análisis la frontera de eficiencia no incluye a las unidades virtuales, aquellas que se forman de la combinación de las unidades de decisión eficientes, el análisis FDH es menos exigente que el DEA, debido a que el DEA requiere de un mayor número de unidades de decisión eficientes.

El único supuesto del FDH es que las entradas y/o salidas pueden disponer libremente de ellos, siendo posible con la misma tecnología de producción, analizar cambios en las variables de salidas o resultado manteniendo el nivel de insumos, o analizar cambios en los insumos manteniendo las salidas o resultados en el mismo nivel. Este supuesto garantiza la existencia de un FDH continua, o frontera de posibilidades de producción, para cualquier muestra de los resultados de producción. Por lo tanto, el análisis FDH proporciona una herramienta intuitiva que se puede utilizar para identificar las mejores prácticas en el gasto público y para evaluar cómo los gobiernos de los países van avanzando en comparación con estas mejores prácticas.

Varios estudios han utilizado el análisis FDH para evaluar la eficiencia del gasto público, donde uno de los estudios de Vanden Eeckhaut, Tulkens y Jamar (1993) han tratado de establecer la eficiencia relativa del gasto de los municipios en Bélgica. En esta investigación se realizó una comparación de los resultados del análisis FDH con los resultados de DEA, y concluyen que el supuesto de convexidad impuesta por DEA distorsiona los resultados de análisis de la eficiencia, esto se debe a que esta hipótesis implica definir la frontera considerando cualquier combinación lineal de dos unidades de decisión observadas también pertenecen al mismo, sin embargo la eliminación de este supuesto consiste en añadir restricciones que definen a las variables generando una frontera escalonada.
Existen muchos intentos o trabajos que han estimado la eficiencia relativa del gasto público en diversos países, por ejemplo Afonso, Schuknecht y otros (2003) analizaron una medición del desempeño del sector público y la eficiencia de los sectores públicos a través de indicadores socioeconómicos de 23 países industrializados de la OCDE (Organización para la Cooperación y el Desarrollo Económicos). Concluyendo que los países con sector público pequeño muestran una distribución más equitativa del ingreso, reportando indicadores significativamente más altos que los países con sectores públicos medianos o grandes.

Roberto Machado (2006), realizo un estudio para el Banco Interamericano de Desarrollo donde realiza un análisis comparativo regional acerca de la eficiencia del gasto público tanto a nivel agregado como a nivel del gasto social, el gasto en educación y el gasto en salud para ocho países de Centro América (Belice, Costa Rica, El Salvador, Guatemala, Honduras, Nicaragua, Panamá y República Dominicana). Este estudio demuestra que para mejorar el desempeño del sector público se debe poner énfasis en aumentar los recursos disponibles o en mejorar la eficiencia en su utilización. Concluye que países como Guatemala, El Salvador y República Dominicana deben incrementar su gasto debido a que existe falta de recursos; países como Belice, Costa Rica y Panamá deben mejorar en la eficiencia del gasto y Honduras y Nicaragua deberían incrementar sus recursos en gasto y eficiencia en su administración. De esta manera estos países pueden tomar medidas de política para mejorar su situación actual.

Un estudio de investigación evalúa la eficiencia del sector público a través del análisis envolvente de datos, el mismo que fue realizado por Afonso Antonio, Schuknecht Ludger, Tanzi Vito (2006), tuvo como objetivo estudiar la eficiencia relativa del sector público de nuevos estados que integran la Unión Europea con las economías emergentes de Asia, se desarrolló bajo un método no paramétrico en términos de la variable mencionada, así mismo el método es usado para la medición del desempeño a través de diversos indicadores para estimar la eficiencia relativa. Este estudio muestra como resultado que la eficiencia del gasto en los nuevos miembros de la Unión Europea muestra un rango intermedio debido a los países emergentes asiáticos que obtienen los mejores resultados en la administración, capital humano, estabilidad económico y crecimiento. Por otro lado se presenta una estimación del modelo de Tobit, sugiere que la seguridad de los derechos de propiedad, PIB per cápita, competencia de los funcionarios públicos y nivel de educación de las personas afecta positivamente la eficiencia del gasto.

En el artículo científico de Sanjeev Gupta y Marijin Verhoeven (1999) analizaron la eficiencia del gasto público en educación y salud en 37 países de África en 1984 a 1995 en comparación con los países de Asia y Occidente a través de la metodología de FDH. Ellos muestran que África tiene un puntaje relativamente bajo 
en términos de eficiencia, mientras que, en promedio, presenta un mayor gasto en educación como porcentaje del PIB de los países de Asia y el Hemisferio Occidental. En el último período de tiempo (1992-1995), el gasto en educación en África un promedio de $4,0 \%$ del PIB, frente al 3,2\% del PIB en Asia y 3,4\% del PIB en el Hemisferio Occidental. Sin embargo, las ineficiencias observadas en África no están relacionados con el nivel de gasto público, pero esto se debe al contexto de estos países que muestran salarios relativamente altos del gobierno (en el caso del gasto en educación) y la asignación intrasectorial de recursos gubernamentales que generan la ineficiencia. El estudio sugiere que debe existir precaución en el aumento presupuestario en las asignaciones para la educación y la salud, y que la atención adecuada se debe dar a la mejora de la eficiencia del gasto existente.

Existen estudios anteriores que muestra la medición de la eficiencia en diversos sectores entre los más importantes son en el sector educación y salud. Uno de los trabajos en América Latina en el sector educación fue realizado por José Luis Pereyra A. (2002), donde demuestra que un mayor gasto en educación no necesariamente significa aumentar su eficiencia, de acuerdo a los resultados obtenidos, el Perú ha logrado un incremento real del gasto en educación durante la última década, sin embargo ello no significa que tal gasto haya sido eficiente respecto al desempeño de otros países de América Latina de bajos ingresos. Llega a la conclusión de establecer una estrategia de financiamiento del gasto en educación con la participación del sector privado que fomente la inversión en los niveles de educación primaria y secundaria principalmente y adoptarse indicadores estandarizados y claramente establecidos sobre el desempeño de los colegios públicos y privados que ayude tanto en las decisiones de los padres de familia como la labor de evaluación educativa.

Para el caso de gobiernos locales existen pocos estudios sobre la evaluación de la eficiencia del sector público, hallándose entre ellos el trabajo de Afonso y Fernandes (2003), en el cual analiza la eficiencia del gasto en gobiernos locales en Portugal utilizando la técnica no paramétrica para la estimación de frontera de producción conocido como Free Disposal Hull (FDH), a base de un índice de desempeño para 51 municipios portugueses situados en la región de Lisboa y Valle del Tajo (RLVT) demostrando que son relativamente ineficiente debido a que se podría obtener el mismo nivel de producción local empleando cerca de un 39\% menos recursos.

Respecto a los estudios explicados anteriormente, se tiene que un mayor gasto no necesariamente ocasiona mejores resultados, existen evidencias empíricas que países con mayor presupuesto no obtienen los resultados esperados debido a la ineficiencia en el uso del gasto público. Así mismo en el Perú de manera constante se presenta un contexto idéntico generando grandes pérdidas económicas que podrían ser considerables para nuestra sociedad, donde sus ciudadanos son los más vulnerables debido a los servicios que brinda el estado peruano.

\section{Materiales y Método}

La investigación aplicada ha sido de carácter explicativo orientado a determinar la relación de causa y efecto entre el gasto de inversión pública con recursos del Canon Minero y el desempeño del sector público en una muestra de cinco regiones mineras del Perú, Ancash, Arequipa, Cajamarca, Moquegua y Pasco, en el rango de años del 2004-2015, aplicando el modelo de Free Disposal Hull (FDH) o de frontera de producción, lo que caracteriza la investigación de tipo transversal-no experimental; tomando en cuenta los principales indicadores socioeconómicos de la base de datos de fuentes oficiales, principalmente de la base de datos digital del Instituto Nacional de Estadística e Informática y de los Anuarios Estadísticos del Ministerio de Energía y Minas del Perú, para varios años.

Se propone la hipótesis que las regiones políticas que han contado con recursos financieros provenientes del Canon Minero y Regalías Mineras y que fueron destinados al gasto de inversión pública, han tenido un desempeño homogéneo en su bienestar medido en términos de sus indicadores económicos y sociales.

En primer lugar se desarrolló el índice general de desempeño del sector público (IDSP) como el indicador out put, el cual está compuesto para esta investigación por 7 indicadores de desempeño público (Afonso, Schuknecht y Tanzi,2005), los cuatro primeros hacen referencia a los resultados obtenidos en las áreas administrativas, educación, salud e infraestructura pública que son considerados como indicadores de oportunidad debido a que tratan de reflejar la calidad de las interacciones entre las políticas fiscales y los procesos de mercado y los otros tres indicadores son las tareas "musgravianas" del gobierno, equidad, estabilidad y reducción de la pobreza. El indicador total o general de desempeño del sector público combina estos siete indicadores que se muestra en la siguiente tabla de indicadores 1 :

Tabla 1. Indicadores utilizados para el IDSP.

\begin{tabular}{|c|c|c|}
\hline Equidad & $\begin{array}{l}\text { Coeficiente de Gini } \\
2014\end{array}$ & $\%$ \\
\hline \multirow[t]{2}{*}{ Estabilidad } & $\begin{array}{l}\text { Coeficiente Variación } \\
\text { tasa crec. PBI 2004- } \\
2015\end{array}$ & Índice \\
\hline & Inflación 2004-2015 & Variación \% \\
\hline Pobreza & $\begin{array}{l}\text { Índice Desarrollo } \\
\text { Humano }\end{array}$ & Índice \\
\hline \multirow{3}{*}{ Educación } & Tasa alfabetismo & $\begin{array}{l}\% \text { de la } \\
\text { población }\end{array}$ \\
\hline & $\begin{array}{l}\text { Tasa neta de } \\
\text { matrícula escolar } \\
\text { primaria }\end{array}$ & $\begin{array}{l}\% \text { población de } \\
6 \text { a } 11 \text { años }\end{array}$ \\
\hline & $\begin{array}{l}\text { Tasa neta de } \\
\text { matrícula escolar } \\
\text { secundaria }\end{array}$ & $\begin{array}{l}\% \text { población de } \\
12 \text { a } 16 \text { años }\end{array}$ \\
\hline
\end{tabular}


Continuación de Tabla 1

\begin{tabular}{|c|c|c|}
\hline \multirow{3}{*}{ Salud } & $\begin{array}{l}\text { Esperanza de vida al } \\
\text { nacer }\end{array}$ & $\mathrm{N}^{\circ}$ años edad \\
\hline & $\begin{array}{l}\text { Tasa mortalidad } \\
\text { infantil }\end{array}$ & $\begin{array}{l}\text { 1/1000 niños } \\
\text { hasta los } 5 \text { años }\end{array}$ \\
\hline & \begin{tabular}{|l|} 
Tasa mortalidad \\
materno \\
\end{tabular} & $1 / 100000$ \\
\hline \multirow{3}{*}{$\begin{array}{l}\text { Desempeño } \\
\text { Económico }\end{array}$} & \begin{tabular}{|l|} 
Tasa crec. anual PBI \\
$2004-2015$ \\
\end{tabular} & $\%$ \\
\hline & \begin{tabular}{|l|} 
Tasa desempleo $(\%)$ \\
2004-2015
\end{tabular} & $\%$ \\
\hline & $\begin{array}{l}\text { PBI per cápita 2004- } \\
2015\end{array}$ & Soles constantes \\
\hline \multirow{8}{*}{ Infraestructura } & $\begin{array}{l}\text { Líneas de telefonía } \\
\text { fija (\% hogares) }\end{array}$ & $\%$ hogares \\
\hline & $\begin{array}{l}\text { Líneas de telefonía } \\
\text { pública (\% hogares) }\end{array}$ & $\%$ hogares \\
\hline & $\begin{array}{l}\text { Servicio de Internet } \\
(\% \text { hogares })\end{array}$ & $\%$ hogares \\
\hline & $\begin{array}{l}\text { Población con luz } \\
\text { eléctrica en su hogar } \\
(\%)\end{array}$ & $\%$ hogares \\
\hline & $\begin{array}{l}\text { \% Hogares que se } \\
\text { abastecen de agua } \\
\text { mediante red pública }\end{array}$ & $\%$ hogares \\
\hline & \begin{tabular}{|l}
$\%$ Población \\
con servicios \\
de saneamiento \\
mejorados \\
\end{tabular} & $\begin{array}{l}\% \text { de la } \\
\text { población }\end{array}$ \\
\hline & $\begin{array}{l}\text { \% Hogares que } \\
\text { tienen red pública de } \\
\text { alcantarillado }\end{array}$ & $\%$ hogares \\
\hline & $\begin{array}{l}\text { \% Locales escolares } \\
\text { públicos con servicios } \\
\text { de agua, desagüe y } \\
\text { electrificación }\end{array}$ & $\%$ local escolar \\
\hline
\end{tabular}

$\mathrm{Y}$ en el caso del indicador in put, se han utilizado los datos de las transferencias del canon y regalías mineras a las regiones respectivas, todo esto medido en relación al producto bruto interno de cada región, así también en relación al total de la población involucrada en cada región política, y en ambos casos se obtuvo el promedio anual de la serie 2004-2015. Este grupo de data es procesada en el modelo FDH.

El empleo de la metodología del FDH (Free Disponsal $H u l l)$, como técnica no paramétrica, permitió estimar una frontera de posibilidades de producción formada por la mejor combinación de resultados dentro de una muestra de las cinco regiones analizadas.

De acuerdo a este método, se debe identificar la región con el máximo IDSP de la muestra (B) del gráfico 1, el cual automáticamente estará sobre la Frontera. Todas las otras regiones que tengan un nivel de gasto superior a él, estarán por debajo de la frontera. Luego se identifica la región con el máximo IDSP que tenga menor gasto que B (A). Todas las regiones que tengan un nivel de gasto superior a $\mathrm{A}$ e inferior a $\mathrm{B}$, también estarán por debajo de la Frontera. A continuación, se identifica al país con el máximo IDSP con menor gasto que A, y así sucesivamente. Se continúa con este proceso hasta llegar a la región con menor nivel de gasto, que por definición también estarán por debajo de la frontera, debido a que no existirá ninguno que logre un mayor IDSP con un menor nivel de gasto.

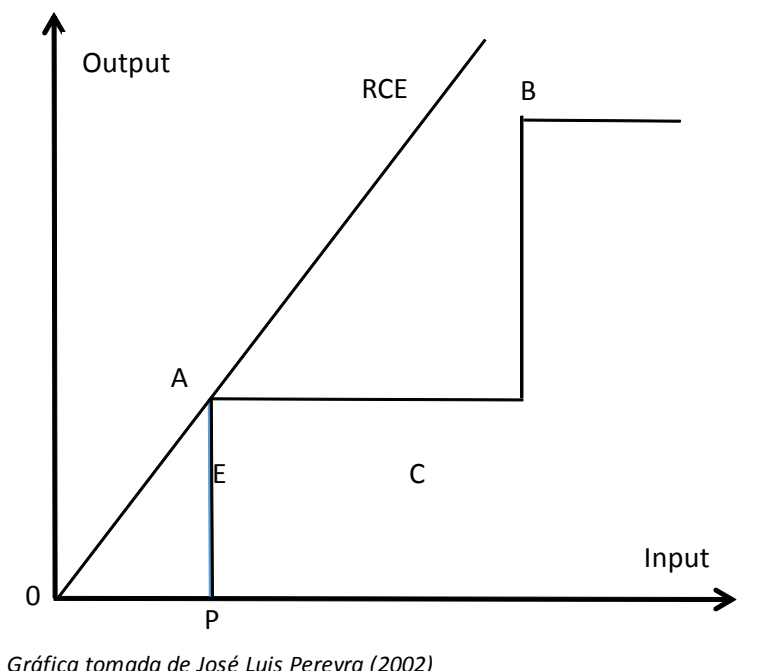

Gráfico 1. Frontera de Posibilidades de Producción.

Al final, la Frontera unirá todas estas observaciones y tendrá una forma escalonada de modo que obtenga las siguientes relaciones, de acuerdo a Machado (2006):

$$
I D S P=f(\text { gasto })=\left\{\begin{array}{c}
I D S P_{A} ; \text { gasto }_{A} \leq \text { gasto } \\
I D S P_{B} ; \text { gasto }_{B} \leq \text { gasto }_{\text {gasto }}< \\
\ldots \ldots \ldots \ldots \ldots . \\
I D S \text { gasto }_{G} ; \text { gasto }_{G} \leq \text { gasto }_{\text {gasto }}<\text { gasto }_{F} \\
0 ; \text { gasto }_{\text {gasto }}
\end{array}\right\}
$$

Donde los subíndices indican las regiones, el gasto está expresado como porcentaje del PIB regional, de esta manera se puede calcular los índices de eficiencia insumos y eficiencia resultados. El primero se relaciona con la distancia horizontal entre la observación de la región y la frontera. La segunda se relaciona con la distancia vertical. Como la derivación de la frontera se basa en la comparación de las observaciones gasto-desempeño de las regiones, ésta dependerá de la muestra de las regiones que se deben considerar para la evaluación. No obstante se pueden encontrar regiones sobre la frontera, lo que no significa que no exista nada que mejorar en cuanto a la eficiencia del gasto en ellos. Esto quiere decir que en comparación a las otras regiones, estos son los más eficientes para su nivel de gasto público. 
La frontera eficiente viene dada por la curva PAB, en tanto que $\mathrm{C}$ representa un punto ineficiente de producción. De esta forma si se sigue la trayectoria EC incrementaríamos insumos (gasto de inversión pública), pero el nivel de producción (IDSP) se mantendría invariable, por lo cual es posible "desechar sin costo" todo incremento adicional de insumos, lo cual le da a nuestra frontera PAB una forma escalonada a diferencia de una frontera de producción con retornos constantes a escala definidas por la recta RCE.

\section{Resultados}

En cada caso, se consideran los datos de los siete indicadores relevantes para construir los IDSP, los cuales son normalizados de modo que al valor promedio de las 5 regiones se le asigna el valor de uno. En el caso de variables donde un menor valor indica un mejor desempeño (por ejemplo, la tasa de desempleo) se toma el valor inverso; luego se procede a un nuevo ajuste de los índices resultantes, asignándole el valor de uno al promedio de la muestra. $\left({ }^{2}\right)$

El IDSP general se obtiene del promedio de los siete índices, tratándose de evaluar el desempeño de la inversión pública financiada con recursos del canon, se le ha otorgado un alto peso o ponderación (50\%) al IDSP en infraestructura; y el otro $50 \%$ se ha distribuido entre los otros seis indicadores de manera equitativa, esto debido a que los recursos del canon deberían ser destinados en su mayor proporción a inversión en infraestructura.

La tabla 2 presenta los resultados procesados en cada uno de los siete índices de desempeño y el IDSP general.

Los resultados dan cuenta que las regiones mineras de Cajamarca, Arequipa y Pasco han logrado un desempeño aceptable en términos de desarrollo económico y estabilidad, pero en materia de equidad solo la región de Arequipa ha dado muestra de logros destacado. En cuanto a los IDSP en educación, salud y en infraestructura destacan las regiones mineras del sur, principalmente Moquegua, lo que ha permitido también conseguir los mejores resultados en el indicador de reducción de pobreza. Con todo ello las regiones del Arequipa y la de Moquegua han mostrado hasta ahora los mejores logros relativos en los indicadores trabajadas y con ello en el IDSP general, como puede observarse en el gráfico 2 .

Del otro lado, se procesó la información de las transferencias gubernamentales bajo el concepto de Canon minero y regalías mineras a las cinco regiones de nuestra muestra, indistintamente haya ido al gobierno regional, provincial o distrital, esto para fines de gasto de inversión pública de impacto regional. Estos recursos o fondos públicos fueron normalizados en términos de su PBI regional y también en términos per cápita.

Los resultados son totalmente distintos, por ejemplo, la región de Moquegua ha contado con un presupuesto con fuente de canon y regalías mineras hasta seis veces de lo que ha contado la región de Arequipa en términos per cápita, sin embargo, ambos tienen similar o cercano resultado en el nivel de desempeño o logro reflejado en el IDSP general, lo que demuestra de un lado que ha existido un alto grado de ineficiencia en el uso de insumos (recursos del canon) por parte de la región de Moquegua o de otra forma, ha existido una alta eficiencia en el uso de insumos por parte de la región de Arequipa, tal como se puede observar en la tabla 3 y gráfico 3.

Destaca también la importante participación del recurso del canon y regalías mineras en términos del PBI regional manejado por la región de Ancash (4.5\%), equivalente a tres veces lo manejado por la región de Arequipa (1.57\%), pero los resultados no se reflejan en el IDSP general, 2.06 Ancash y 2.66 Arequipa, lo que demuestra una ineficiencia en el uso de insumos en la región de Ancash. Así también, y en términos del PBI regional, lo observado en la región de Cajamarca $(3.47 \%)$, es que ha contado con un presupuesto con fuente del canon y regalías mineras equivalente a más de dos veces de lo manejado por la región de Arequipa, pero solo ha logrado la mitad o cercano a ello en términos del IDSP general, 1.42 Cajamarca y 2.66 Arequipa, no olvidemos que la región de Cajamarca sigue ocupando el primer puesto de nivel de pobreza en el Perú, a pesar de contar con importantes recursos como el señalado en este estudio.

Finalmente, integrando lo anteriormente expuesto, se presenta la frontera FDH (Free Disponsal Hull), tomando en cuenta los mismos datos de la tabla 3, lo que permitió estimar una frontera de posibilidades de producción formada por la mejor combinación de resultados dentro de una muestra de las cinco regiones analizadas, la misma que se presentan en los gráficos 4 y 5 .

Los resultados no hacen otra cosa que confirmar lo hasta ahora obtenido. En efecto, en términos de logros conseguidos en el IDSP general, la región de Arequipa ha dado muestra de haber obtenido un mejor desempeño en los indicadores analizados en relación a los indicadores de recursos disponibles provenientes del canon y regalías mineras.

Se corrobora que las regiones de Moquegua y Ancash han podido y debido haber logrado los mismos o cercanos desempeños en el IDSP general que la región de Arequipa con una menor asignación de presupuesto del canon minero, de ahí que su posición es al interior y extremos derechos de la frontera FDH.

Igualmente se confirma, entre otras cosas, que las regiones de Cajamarca y Pasco han podido y debido haber logrado los mismos o cercanos desempeños en el IDSP general que la región de Arequipa, teniendo las tres regiones similares o cercanos montos en el presupuesto de canon y regalías mineras.

2La fórmula utilizada es (Obs - Min) / (Max - Min), donde Obs es el valor observado, min el valor mínimo, y Max el valor máximo, tomado de Machado (2006) 
Tabla 2. Índices de desempeño del sector público normalizados

\begin{tabular}{|l|c|c|c|c|c|c|c|c|}
\cline { 2 - 9 } \multicolumn{1}{c|}{} & IDSP Equidad & $\begin{array}{c}\text { IDSP } \\
\text { Estabilidad }\end{array}$ & $\begin{array}{c}\text { IDSP Des. } \\
\text { Econ. }\end{array}$ & $\begin{array}{c}\text { IDSP } \\
\text { Educación }\end{array}$ & $\begin{array}{c}\text { IDSP } \\
\text { Salud }\end{array}$ & $\begin{array}{c}\text { IDSP } \\
\text { Infraest. }\end{array}$ & $\begin{array}{c}\text { IDSP } \\
\text { Pobreza }\end{array}$ & $\begin{array}{c}\text { IDSP } \\
\text { General }\end{array}$ \\
\hline Ancash & 2.2727 & 1.9550 & 1.5908 & 2.0149 & 1.9036 & 2.2367 & 1.5370 & 2.0579 \\
Arequipa & 3.0000 & 2.0000 & 1.5755 & 2.3333 & 2.6394 & 2.9531 & 2.6443 & 2.6593 \\
Cajamarca & 1.0000 & 2.8035 & 1.9836 & 1.1140 & 1.4533 & 1.2861 & 1.0000 & 1.4226 \\
Moquegua & 1.7273 & 1.5100 & 1.6933 & 2.9535 & 2.8171 & 2.7373 & 3.0000 & 2.5104 \\
Pasco & 2.6364 & 1.9284 & 1.9469 & 2.0162 & 1.5788 & 1.2030 & 1.2793 & 1.5503 \\
\hline
\end{tabular}

Fuente: Base de datos digital INEI, MEM, varios años. Elaboración: Propia
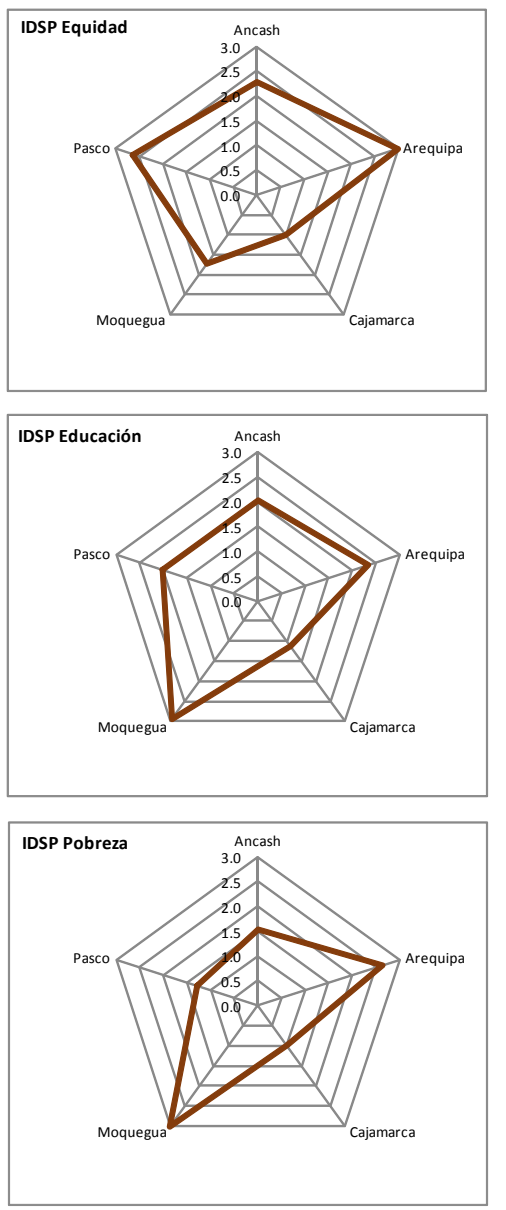
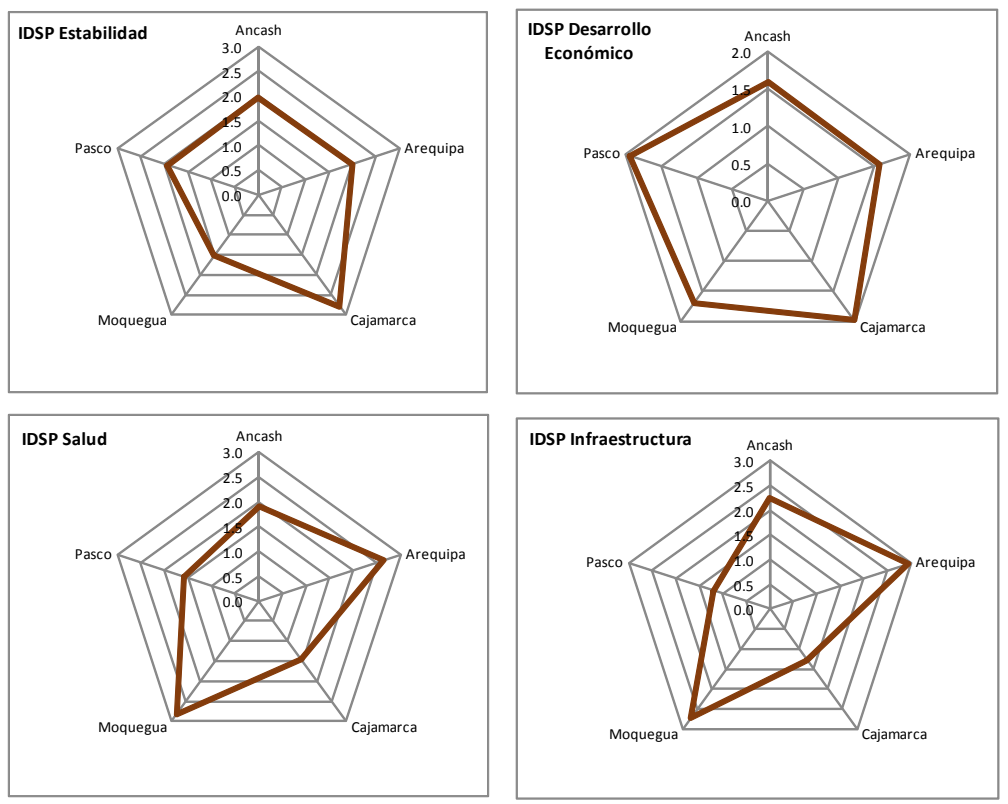

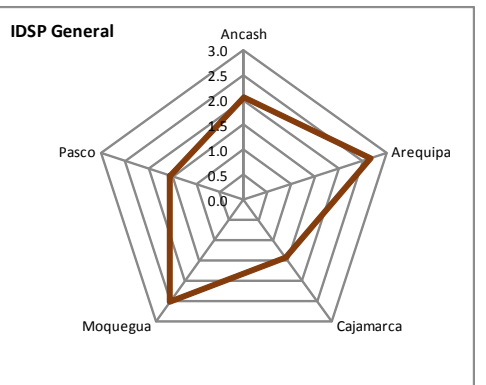

Gráfico 2. Resultados en el desempeño para cada IDSP y el General

Tabla 3. Recursos de canon y regalías mineras

\begin{tabular}{|c|c|c|c|}
\hline & \multicolumn{3}{|c|}{ Promedio 2004 - 2015} \\
\hline & $\begin{array}{c}\text { Canon / } \\
\text { PBI }\end{array}$ & $\begin{array}{c}\text { Canon } \\
\text { Per-Capita } \\
\text { (Soles) }\end{array}$ & $\begin{array}{c}\text { IDSP } \\
\text { General }\end{array}$ \\
\hline \multirow{5}{*}{$\begin{array}{l}\text { Ancash } \\
\text { Arequipa } \\
\text { Cajamarca } \\
\text { Moquegua } \\
\text { Pasco }\end{array}$} & $4.50 \%$ & 671.16 & 2.06 \\
\hline & $1.57 \%$ & 316.17 & 2.66 \\
\hline & $3.47 \%$ & 257.44 & 1.42 \\
\hline & $3.81 \%$ & $1,988.34$ & 2.51 \\
\hline & $2.96 \%$ & $\ldots 606.75$ & 1.55 \\
\hline
\end{tabular}

Fuente: Base de datos digital INEI, MEM, varios años.

Elaboración: Propia 

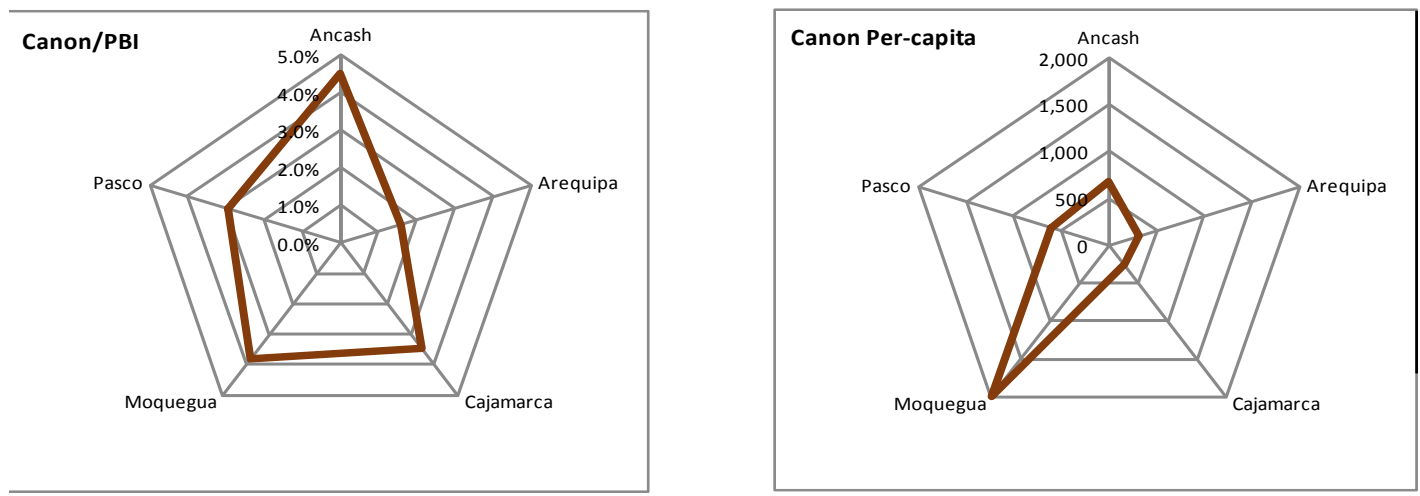

Gráfico 3. Recursos de canon y regalías mineras, Promedio 2004 - 2015

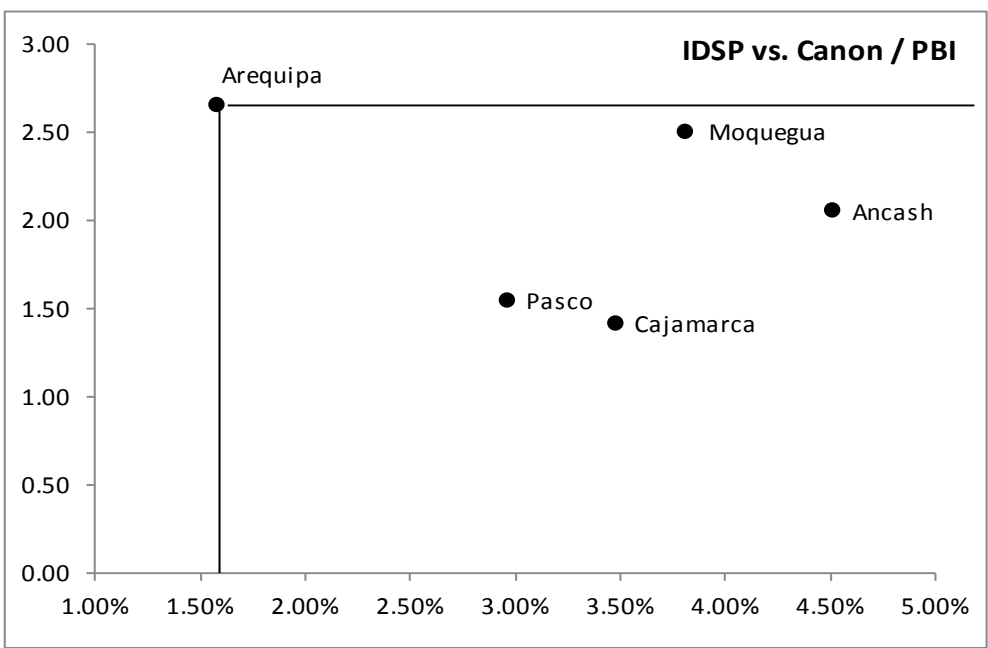

Fuente: Base de datos digital INEI, MEM, varios años (2004-2015) Elaboración: Propia

Gráfico 4. Frontera de Posibilidades de Producción IDSP y Canon/PBI

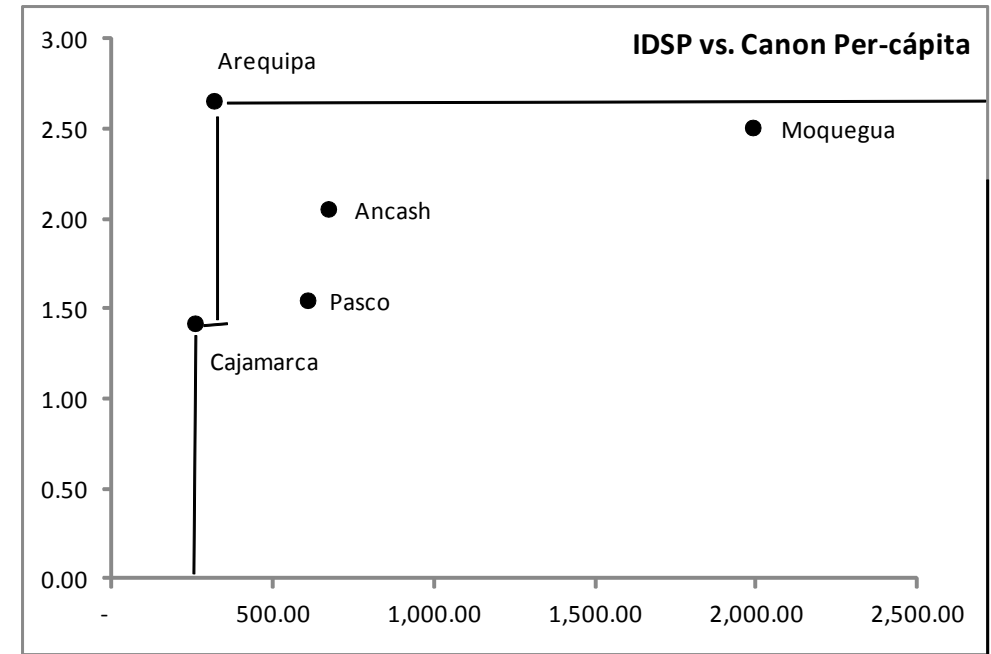

Fuente: Base de datos digital INEI, MEM, varios años (2004-2015)

Elaboración: Propia

Gráfico 5. Frontera de Posibilidades de Producción IDSP y Canon Per cápita 


\section{Conclusiones}

Como resultados de la presente investigación, ha quedado demostrado que las regiones políticas que han contado con recursos financieros provenientes del Canon Minero y que fueron destinados al gasto de inversión pública, no han tenido un desempeño homogéneo en su bienestar medido en términos de sus indicadores económicos y sociales.

Por el contrario, tenemos regiones que teniendo menos recursos financieros ha sido más eficientes en lograr mejores resultados económicos y sociales, en comparación a otras regiones que contando con más recursos financieros han conseguido relativamente menos resultados o desempeño económico y social. En este último caso, ni siquiera está siendo lo suficientemente útil para erradicar los niveles y del ranking de la pobreza de la región.

En resumen, no necesariamente más recursos financieros proveniente de la renta del recurso natural está significando más desarrollo de la región, salvo excepciones, son otras consideraciones que deben investigarse, que estarían determinando las decisiones de políticas públicas sobre en que invertir los recursos públicos (teoría de la elección pública), la participación efectiva de la ciudadanía, en la capacidad gerencial de los recursos humanos calificados, y por último, en la ética durante la decisión y ejecución del gasto de inversión pública.

\section{Literatura citada}

Afonso, A., Schuknecht, L. y Tanzi, V. (2003). "Public Sector Efficiency: An International Comparison" Working Paper No. 242 - European Central Bank.

Afonso, A. y Fernandes, Sonia (2003). "Efficiency of Local Government Spending: Evidence for the Lisbon Region"

Afonso, A., Ebert, W., Schuknecht, L. and Thöne, M. (2005). "Quality of public finances and growth," European Central Bank, Working Paper n. 438.

Afonso, A., Schuknecht, L. y Tanzi, V. (2006). "Public Sector Efficiency: Evidence For New EU Member States and Emerging Markets" Working Paper No. 242 European Central Bank.

Afonso, A. y Aubyn, St. (2004). Non - parametric Aproaches to Education and Health Expenditure Efficiency in OECD Countries

Ahn T., Charnes, W. y Cooper, W. (1988). Using Data Envelopment Analysis to Measure the Efficiency of Not - for Profit Organizations: A Critical Evaluation Comment. Managerial and Decisison Economics. Vol 9. No. 3, pp. $251-253$.

Arrow, K. J. (1974). Elección social y valores individuales Ed. Ministerio de Economía y Hacienda.
Asmad, U., Palomino, D., Tam, M. y Zambrano, G. (2004). Una aproximación a la alfabetización matemática y científica de los estudiantes peruanos de 15 años Resultados del Perú en la evaluación Internacional PISA. Unidad de Medición de la Calidad. Ministerio de Educación del Perú. Documento de trabajo No. 10.

Banker, R., Charnes, A. y Cooper, W. (1984). Some Models for Estimating Technical and Scale Inefficiencies in Data Envelopment Analysis. Managerial and Decisison Economics. Vol. 30. No. 9, pp. 1078 - 1092.

Becker, G. (1983). El capital Humano. Alianza Editorial 1983.

Cleary, E. (2006). El Benchmarking desde el sector privado al sector público "Apuntes sobre su aplicación en el sector público en América Latina”,

De Borger, B. y Kerstens, K. (1996). "Cost efficiency of Belgian local governments: A comparative analysis of FDH, DEA, and econometric approaches", Regional Science and Urban Economics, 26, 145-170.

De Borger, B. and Kerstens, K. (2000). "What is known about Municipal Efficiency?" in: Blank, Jos L.T. (ed), Public Provision and Performance: contributions from efficiency and productivity measurement, Amsterdam, North-Holland, 299-330.

Geys, B and Moesen, W. (2008). "Measuring Local Government Technical (In) efficiency: An Application and Comparison of FDH, DEA and Econometric Approaches"

Gupta Sanjeev and Verhoeven Marijin (2001). "The Efficiency of Government Expenditure Experiences from Africa” Journal of Policy Modeling 23: 433 - 467.

Joumard, I., Kongsrud, P., Nam, Y.-S. and Price, R (2004). "Enhancing the Effectiveness of Public Spending: Experience in OECD Countries", OECD Economics Department Working Paper 380.

Machado, R. (2006). “¿Gastar más o Gastar mejor? La eficiencia del Gasto Público en América Central y República Dominicana" Serie de Estudios Económicos y Sectoriales del Banco Interamericano de Desarrollo.

Murillo-Zamorano, L. (2004). "Economic Efficiency and Frontier Techniques" Journal of Economic Surveys, 18 (1), $33-77$.

Pereyra, A. José Luis (2002). "Una medida de la eficiencia del gasto público en educación: Análisis FDH para América Latina” Estudios Económicos No. 237 Banco Central de Reserva del Perú.

Samuelson, Paul A. (2006) Economía Ed. McGraw Hill.

Stiglitz, J. E. N. (1988). Economía del Sector Público Ed. Antoni Bosch.

Straface, F. (2003). "Latin American and Caribbean Award for Innovations in Government". International Development Bank. 
Tam, M. (2008). "Una aproximación a la eficiencia técnica del Gasto Público en Educación en las regiones del Perú" Consorcio de Investigación Económica y Social.

Tandon, A. (2005). "Measuring Efficiency of Macro Systems: An Application to Millennium Development Goal Attainment" Economics and Research Department Working Paper No. 66 - Asian Development Bank.

Tanzi, V. (2008) "The Economic Role of the State in the $21^{\text {st }}$ Century" Organization for Economic Cooperation and Development.

Vanden Eeckaut P., Tulkens, H. and MA Jamar (1993).

"Cost Efficiency in Belgian Municipalities." in Fried HO and SS Schmidt (eds.) the Measurement of Productive Efficiency: Techniques and Applications, Oxford U.K.: 300-334. 